\title{
Adaptive Deep Learning based Cryptocurrency Price Fluctuation Classification
}

\author{
Ahmed Saied El-Berawi ${ }^{1}$ \\ Computer Networks and Datacenter \\ Arab Academy for Science \\ Technology and Maritime and \\ Maritime Transport \\ Alexandria, Egypt
}

\author{
Mohamed Abdel Fattah Belal ${ }^{2}$ \\ Faculty of Computers and Artificial \\ Intelligence, Helwan University \\ Cairo, Egypt
}

\author{
Mahmoud Mahmoud Abd Ellatif ${ }^{3}$ \\ College of Business, University of \\ Jeddah, Saudi Arabia \\ Faculty of Computers and Artificial \\ Intelligence, Helwan University \\ Cairo, Egypt
}

\begin{abstract}
This paper proposes a deep learning based predictive model for forecasting and classifying the price of cryptocurrency and the direction of its movement. These two tasks are challenging to address since cryptocurrencies prices fluctuate with extremely high volatile behavior. However, it has been proven that cryptocurrency trading market doesn't show a perfect market property, i.e., price is not totally a random walk phenomenon. Based upon this, this study proves that the price value forecast and price movement direction classification is both predictable. A recurrent neural networks based predictive model is built to regress and classify prices. With adaptive dynamic features selection and the use of external dependable factors with a potential degree of predictability, the proposed model achieves unprecedented performance in terms of movement classification. A naïve simulation of a trading scenario is developed and it shows a $69 \%$ profitability score a cross a six months trading period for bitcoin.
\end{abstract}

Keywords-Computer intelligence; cryptocurrency; deep learning; market movement; recurrent neural network; timeseries forecasting

\section{INTRODUCTION}

Cryptocurrency, or digital currency, is a virtual currency used for the exchange and transfer of assets. When compared to traditional currencies, which rely on central banking institutions, cryptocurrencies are built on the idea of decentralized control. As a result, a cryptocurrency is used to send money electronically without the involvement of a central or governmental authority. Within the last few years, and due to its uncontrollable and untraceable character, there has been a growing interest in cryptocurrency trading. The industry has grown tremendously for financial transactions and trading throughout the world. It also shows a continuing and growing trend for the near future. The market is expected to rise from 1.8 billion to 2.2 billion USD by 2026 according to https://coinmarketcap.com/charts/" (accessed Jul. 18, 2021). Moreover, when compared to traditional state-issued currencies, cryptocurrencies are extremely volatile. Their exchange rates cannot be assumed to be independently and identically distributed phenomena [1]. In a short period of time, the cryptocurrency sector has experienced exponential development and global popularity. Such increase in popularity received significant media attention, attracting more investors, researchers, regulators, and speculators to the field as promising booming business. Such increased popularity necessitates study into their dynamics and how they affect the financial sector and economies of countries in general.

The following sections discuss in short the intrinsic characteristics of cryptocurrency systems that relate to the problem statement addressed, and what are challenges, gaps and research questions of this work.

\section{A. Cryptocurrency Characteristics}

The value of each cryptocurrency is determined by its volume of transactions and price movements. Moreover, each cryptocurrency has its own ecosystem and operates differently from the other ones in terms of value variations, transaction speeds, usages, and volatility. Such independence makes forecasting cryptocurrencies prices a challenging task.

Another characteristic is that despite the massive bitcoin meltdown at the start of 2018, with a lot of volatility, people's interest in it has remained relatively steady. In response, in recent years, academics have presented a variety of approaches for predicting and modelling the price of cryptocurrencies as well as analyzing the volatility of the crypto market. Also, it is difficult to say exactly what drives the price of cryptocurrencies over time. However, $\mathrm{P}$ Katsiampa used the asymmetric Diagonal BEKK model to investigate the volatility dynamics of four main cryptocurrencies in this extensive analytical work that had been published in 2019 [2]. The conditional variances (the variance of a phenomenon given the value(s) of one or more factors in econometrics) of all four cryptocurrencies are strongly impacted by both prior squared errors and past conditional volatility, according to the study. Furthermore, big news has been proven to affect volatility dynamics. Finally, the crypto industry as a whole is known for its price fluctuations and trading volumes. With the growing interest in cryptocurrencies and their importance in the financial sector, extensive research and forecasting of cryptocurrencies' volatility dynamics is needed [3].

Also, because of the exponential growth in speculative activities, cryptocurrency markets are increasingly vulnerable to price fluctuations and degradation. It's still unclear if the biggest price fluctuations in cryptocurrencies are unpredictable or predictable over time. In other words, is it follows the Efficient Market Hypothesis (EMH) or not? 
According to [4], markets are regarded as totally effective when they follow a random (weak-form) model in which future returns on the basis of prior data cannot be forecasted. Many recent studies have been conducted to investigate the efficiency and volatility characteristics of cryptocurrency prices. According to Palamalai et al. [5] the returns from the top cryptocurrencies show a persistency impact, indicating market inefficiency, i.e., not a random-walk process. Their findings have immediate ramifications for cryptocurrency market speculation. For example, the idea that bitcoin history data retains some degree of predictability about future values is correct. Another approach to figuring out predictability for the bitcoin problem is to see whether external elements may be integrated as additional features. Besides these, there are prediction strategies that are already in-use by traders. Most of them are based on heuristics and empirical conclusions, such as "Engulfing Pattern" and "Evening Star". Furthermore, some studies have also shown that social media sentiment analysis, particularly tweets about trading activities, have considerable prediction potentials, check Fig 1 for factors that may contribute to the cryptocurrency pricing.

Based upon this discussion, prediction of price's value and movement direction (whether goes up or down) is a task that plays a vital role in the cryptocurrency economy. Even though the efficiency of the forecasting models has been improved in recent years, most improvements have been achieved by minimizing the error between predictions and real readings. However, due to high fluctuations rates, predicting the next exact value turned to be not that useful for trading purposes more than predicting whether it will go up or down. However, a review of relevant studies reveals that the majority of research activities have exclusively focused on the forecasting model's accuracy. Nonetheless, it is possible to further address the actual goal (which is increasing trading profitability) by setting the focus on the price movement directions rather than, or with addition to, the price values.

With this goal in hand, this work seeks to address the following research questions: 1) Are cryptocurrency prices predictable? And if so, is that due to inherent features of the pricing data? or, alternatively, because of external influences? 2) Which is more important in terms of profitability: forecasting the price value or predicting the price movement direction (increase or drop)? 3) What additions that deep learning algorithms potentially provide in this regard? Whether it's forecasting the price or deciding the price change's direction?

\section{B. Organization of the Paper}

The rest of the paper is organized as follows: Section II outlines various related and recent work in this new field with emphasis on deep learning models. Section III is devoted to this work; the dataset involved in the study, the features engineering, the proposed model architecture. Section IV shows the experiments setup, findings, and results. Finally, Section $\mathrm{V}$ concludes the study and discusses suggested future work.

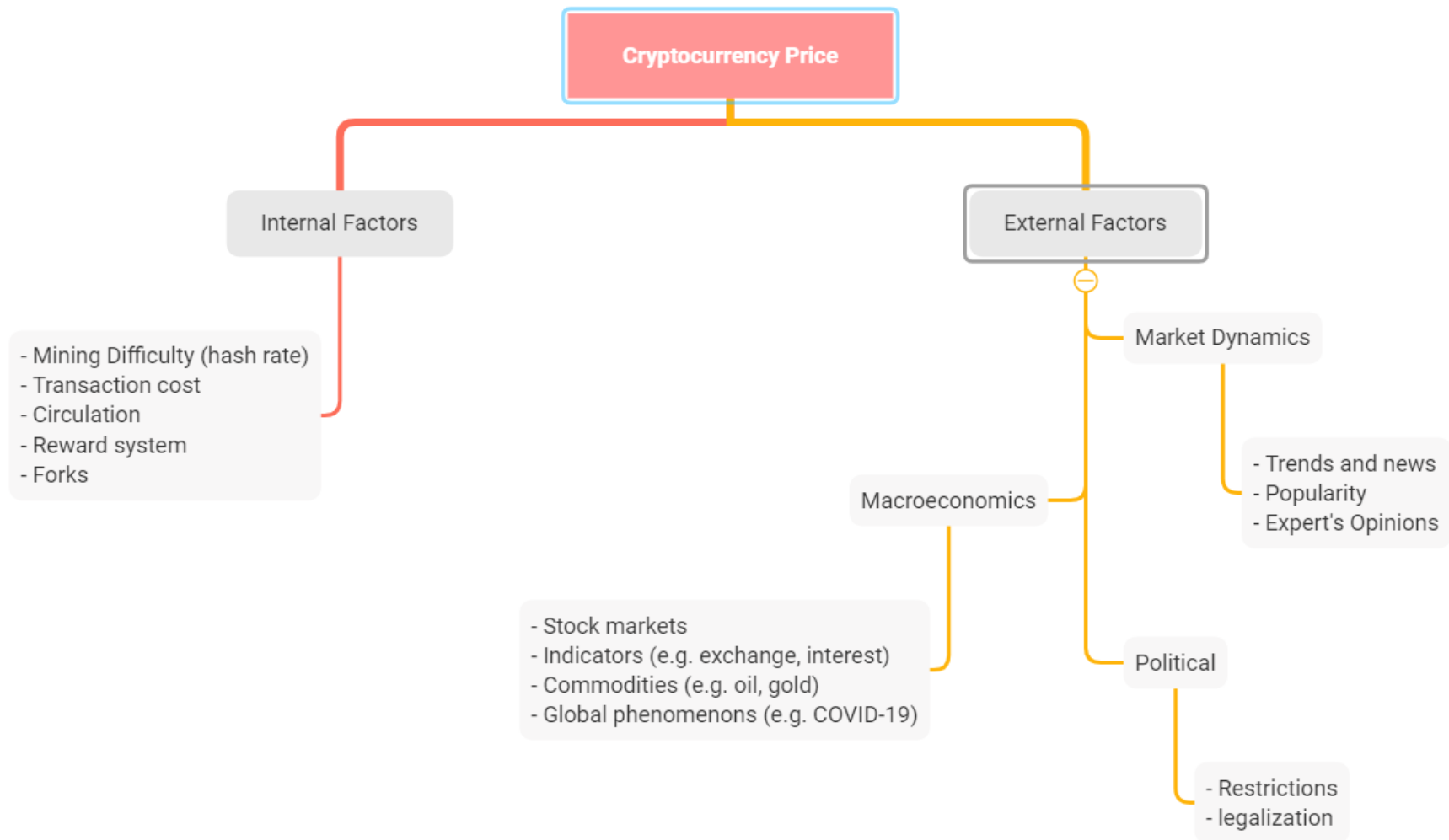

Fig. 1. Factors Affecting Cryptocurrency Prices. 


\section{RELATED WORK}

Over the last several years, there has been a significant surge in research interest in the subject of cryptocurrencies. Since 2015, the number of publications in this field has been increasing, and this trend is expected to continue in 20201, attributed to the growing popularity of cryptocurrencies and their widespread attention as an emerging financial sector. The field of cryptocurrency price prediction is still relatively new, with not much papers having been published; however, it is a very important topic that deserves to be explored because of the impact it is having on the financial system. Also, research trends are mostly focused on forecasting price.

\section{A. Statistical and Traditional Techniques}

A recent study by Palamalai et.al [5] evaluated the weakform market efficiency of a top ten cryptocurrencies. They used random-walk testing with parametric and non-parametric methods which are resistant to "unknown structural breakdowns" and "asymmetric effects". The results confirmed the cryptocurrencies' weak-form inefficiency, and that it refuted the random-walk hypothesis. In essence, the results said that there is potential predictability within the cryptocurrency trading process. Their findings were consistent with which earlier research in 2017 (Hayes et al [3]) that demonstrated the impact of external factors on cryptocurrency predictability.

Holt-Winters exponential smoothing is a classic linear method for time-series forecasting that is commonly used. It splits the input data into several trend components. These components are used to forecast targets that have seasonal characteristics. Peng [6] argued that such traditional linear methods cannot be used to accurately predict cryptocurrency prices because they lack seasonality.

Chu et.al, [1] developed a GARCH method-based models. They investigated the log returns of exchange rates. The maximum likelihood method was used to fit the data. The models were evaluated based on several criteria, and the bestfitting models with the best forecasting performance were selected. Recently, Malladi et.al, [7] investigated the relationships between both Bitcoin and Ripple renderings and volatility, the authors used the Autoregressive Mobiles Average Exogenous Input (ARMAX), the Autoregressive Generalized Conditionally Heteroscedastic (GARCH) model, the Vector Autoregression (VAR) model, and Granger causality tests and showed that the Bitcoin crash of 2018 might have been described by these time series approaches. They also discovered that global stock and gold returns do not cause Bitcoin's returns, but Ripple's returns have a direct impact on Bitcoin pricing.

The impact of the three factors on cryptocurrency value was studied by Hayes et al [3] using cross-sectional data from 66 of the most commonly used cryptocurrencies. The factors are competition levels in the producers' network, production rates of the unit, and the algorithm difficulty used to mine crypto-monetary activity. The authors demonstrated that the three factors have shown a significant impact on price. Blau et.al, [8] used the regression to analyze the impact of the speculative trading, a 5-day bitcoin return, a 5-day volume sale, excess bitcoins, and a volatility estimate on the price of bitcoin of a number of other cryptocurrencies. The results showed that speculative trade has no impact on bitcoin prices. Speculative trade is the trade in future contracts, without obtaining the commodity that underlies it in reality. These traders buy or sell future contracts to re-sell it before their settlement date.

Another early proposition is described in [9] that applied autocorrelation and partial autocorrelation functions. The authors found that the first difference in the Bitcoin exchange rate is a weak-stationary time-series dataset. They created an ARIMA model to forecast future prices. They showed that expost forecasting's mean absolute percent error is 5.36 percent. In this work and in related references [14] it was investigated multiple variables that affecting the values of four cryptocurrencies (Bitcoin, Ethereum, Dash, litecoin, and Monaro). It has been shown that using weekly data and the enhanced Dickey-Fuller unit-root test and bound testing technique from 2010 to 2018. Market exchange, trading volume, and volatility all have an influence on the values of all four cryptocurrencies in the short and long run. This research used ARIMA as a forecast model.

Recently, Akcora et al. [10], [11], [12] have introduced a novel concept of chainlets, or blockchain motifs, to create a complex network of financial interactions on the blockchain that can be used to investigate link between bitcoin risk investing and different blockchain network aspects. Chainlets allow researchers to examine the influence of the blockchain's local topological structure on Bitcoin and Litecoin price development and evolution.

The authors in [13], [14], [15] described a strategy for forecasting changes in Bitcoin and Ethereum values using Twitter data and Google Trends [16]. Twitter is rapidly being utilized as a news source, alerting users about the currency and its rising popularity, affecting purchasing decisions. They discovered that tweet volume is a better predictor of price direction than tweet sentiment, which is always positive regardless of price direction. They were able to properly forecast the direction of price fluctuations using a linear model that takes as input tweets and Google Trends data. Aside from that, Shen et al. [17] argued that the number of tweets from Twitter, rather than Google trends, is a better indication of attention from more knowledgeable investors. They notice that the quantity of tweets has a substantial impact on the trade volume the next day.

Mohapatra et.al, [18] developed a novel KryptoOracle, a unique real-time and adaptable bitcoin price prediction engine based on Twitter emotions. The platform's integrative and modular design includes a Spark-based architecture for durable and fault-tolerant handling of massive amounts of incoming data, as well as real-time natural language processing including sentiment analysis, and an online learning-based prediction approach. Based on the accessible and ever-increasing volume and diversity of financial data, the experimental assessment indicated that the suggested platform may assist in the acceleration of decision-making, the identification of new possibilities, and the supply of more timely insights. 
Bhambhwani et al. [19] used the dynamic ordinary leastsquares approach was used to study the underlying determinants of cryptocurrency prices (Bitcoin, Ethereum, Monero, Litecoin, and Dash). The values of various currencies have been revealed to be influenced by their mining computer power and network connection. In [20], the authors looked into the correlation between Bitcoin market volatility and volatility in other traditional markets including gold, currencies, and stocks. On a daily, weekly, and monthly basis, they used data. Recent data shows a small but positive correlation between changes in Bitcoin volatility and changes in the trade weighted USD currency index volatility, according to correlations and regressions. Furthermore, a greater positive link between Bitcoin volatility and search demands on Bitcoin-related terms on Google has been observed. Furthermore, a VAR-analysis demonstrated that the volatility of the USD currency index, which is the lone driver of future Bitcoin volatility, is to some extent predictive of future Bitcoin volatility.

Vector autoregression method was investigated by Giudici et.al, [21]. The authors introduced partial correlations and correlation networks. The model assisted in determining the dynamics of cryptocurrency prices in various crypto exchange markets and allowed one to understand its correlation with other traditional market prices. The use of VAR correlation networks also allowed for the development of a model for predicting bitcoin price that makes use of the information contained in various correlation patterns among various exchange prices. Dos Santos et al. [22] investigated the dynamic behavior and predictability of bitcoin price dynamics (high and low). They used the fractionally cointegrated vector autoregressive (FCVAR) model to analyze bitcoin and dollar price patterns. The empirical analysis was carried out between January 2012 and February 2018. They compared fractionally cointegrated VAR to various other algorithms, and the results showed that fractionally cointegrated VAR performed better.

In [23] The authors utilized a modified Binary Auto Regressive Tree model to develop a short-term bitcoin price forecasting model (BART). BART is a hybrid method which mixes autoregressive models from the classification and regression trees (C\&RTs). A short-term prognosis was made of the three most important cryptocurrencies, Bitcoin, Ethereum, and Ripple (from 5 to 30 days). The suggested technique has been identified in the prediction of cryptocurrency time slower (fall) and transitional dynamics more accurately than ARIMA-ARFIMA models (change of trend). In RMSE according to the predicted horizon, the suggested model obtained 2,5 to 4,9 percent.

As reported by [15], sentiment analysis can be used as a computational tool to forecast the prices of bitcoin and other cryptocurrencies over various time intervals. The fact that currency prices fluctuate based on people's perceptions and opinions, rather than institutional money regulation, is a key feature of the cryptocurrency market. As a result, examining the relationship between web search and social media is crucial for projecting cryptocurrency prices. Because these social media platforms are used to influence purchasing decisions, this study forecasted the short-term prices of the major cryptocurrencies using Google Trends and Twitter. The study adopts and interpolates a novel multimodal approach to investigate the impact of social media on bitcoin pricing. The findings demonstrate that the psychological and behavioral views of people have a substantial influence on such highly speculative cryptocurrency values.

\section{B. Machine Learning Techniques}

Cryptocurrency is a highly volatile asset. Researchers were inspired to apply DL and ML paradigms to cryptocurrency issues as a result of this. The use of stock market price prediction techniques can help to improve precision. Because of its ability to identify the general trend and fluctuation, machine learning has become one of the most researched approaches in cryptocurrency price prediction in recent years.

One of the early work using machine learning techniques showed in [24], the authors used an SVM, an ANN, linear regression, and logistic regression to predict bitcoin price using blockchain data. The highest price accuracy was $55 \%$ for a NN classifier with two hidden layers, followed by logistic regression and SVM. In addition, the study mentions the use of several tree-based models and K-nearest neighbors in its analysis. In this study, only blockchain data was used for training and prediction, which resulted in limited predictability. According to the findings, using features directly extracted from bitcoin exchanges, such as financial flow features, would likely improve the accuracy of bitcoin price prediction. Hitam et.al, [25] forecasted the cryptocurrency future price using an optimized Support Vector Machine (SVM) based on Particle Swarm Optimization (PSO). The results of forecasting using basic SVM algorithms were found to be unreliable. Meanwhile, PSO's optimized version of SVM shows that it can accurately forecast future cryptocurrency prices, outperforming single SVM algorithms.

Mohanty et al. [26] used LSTM for bitcoin future price prediction. and Twitter data was used to predict public mood. This method selected some key features from the blockchain that had a significant impact on bitcoin demand and supply, and then used them to train a model that improved bitcoin price prediction in the future. The model demonstrated high precision and accuracy. Mittal et al. [27], identified the correlation between bitcoin price and Twitter and Google search patterns using machine learning techniques such as linear regression, polynomial regression, recurrent NN (RNN), and long short-term memory (LSTM)-based analysis. Tweet sentiment analysis performs the worst out of Google Trends, tweet volumes, and tweet sentiments. When LSTM, RNN, and polynomial regression were used to analyze Google Trends and tweet volume, the accuracy of the results improved.

Atsalakis et al, [28] proposed PATSOS, a neuro-fuzzy controller for predicting bitcoin's daily price change trend. The scheme outperformed two other computational intelligence models, the first of which was created using a simpler neurofuzzy approach and the second of which was created using artificial neural networks (ANNs). They also stated that the PATSOS system's performance was stable enough to be used for other cryptocurrencies. 
The results obtained by applying hidden markov models in [29] to the historical cryptocurrency movements, and using LSTM to predict future movements suggested that the proposed approach had the lowest MSE, RMSE, and MAE when compared to traditional time-series prediction models, ARIMA, and conventional LSTM, demonstrating its effectiveness. This hybrid approach's parameters were further optimized using a genetic algorithm. This model did not account for the internal details of bitcoin transactions. As a result, they planned to consider additional features for future work in order to provide more information about the blockchain.

Radityo et.al, [30] showed for the next-day prediction, four variants of ANN were used to use the bitcoin exchange rate (closing price) on the American dollar. They Studied variety of ANN methods to predict the market value of one of the most used cryptocurrencies, Bitcoin. The ANN methods will be used to develop model to predict the close value of Bitcoin in the next day (next day prediction). This study compares four ANN methods, namely backpropagation neural network (BPNN), genetic algorithm neural network (GANN), genetic algorithm backpropagation neural network (GABPNN), and neuro-evolution of augmenting topologies (NEAT). The methods are evaluated based on accuracy and complexity. The result of the experiment showed that BPNN is the best method with MAPE $1.998 \pm 0.038 \%$ and training time $347 \pm 63$ seconds. Maiti et.al, [31] proposed a forecasting approach based on the chaotic co-movement of seven major cryptocurrencies, non-linear forecasting models have been proposed and implemented. For lags 0 and $0-3$ the LSTM outperforms the ANN, while for large lags 0-7 the ANN outperforms the LSTM. Further research confirms that forecasting using variables like volume is ineffective in any case. In [32], As machine learning techniques, the authors used ARIMA, FBProphet, and XGBoosting for time series analysis. Root Mean Square Error (RMSE), Mean Absolute Error (MAE), and R2 are the parameters they used to evaluate these models. They tested these three techniques, but time series analysis revealed that ARIMA was the best model for forecasting Bitcoin price in the crypto market, with an RMSE score of 322.4 and an MAE score of 227.3.

\section{METHODOLOGY}

We use a new approach that achieves two convergent tasks: forecasting the price and classifying the price movement. The intuition is that the focus is not only on the next point's prediction value, but also on the price movement direction whether it's going up or falling down. The determination of whether prices will rise, or fall is critical, particularly for traders. The proposed method focuses on utilizing LSTM layers' ability to extract useful knowledge by learning the internal representation of cryptocurrency features as well as correlation with external factors features. LSTM layers are used to identify short and long-term dependencies between time-series data, which are then used to predict the next point value and answer the question of whether price will fall or rise. Next sections discuss the underlaying hypothesis, the data used in the experiments and the proposed model architecture.
We formulate the price movement direction problem as follows; let $T$ be the time divided into a timestamp $t=T / N$ where $\mathrm{N}$ is number of observations over $T . t$ length maybe in minutes, hours, days, or any interval. Suppose $\mu \in \mathbb{R}$ is the price change value from $t_{k-1}$ to $t_{k}$, where $\mu$ sign indicates the direction of change. The goal is to build a data-driven model to achieve two objectives; 1) classify $\mu_{k}$ sign at time $t_{k}$, and 2) predict the cryptocurrency price value at tine $t_{k}$, given observations of multiple timeseries features within time interval $\left\{t_{0}-t_{k-1}\right\}$.

\section{A. Cryptocurrency Performance Metrics}

For point forecast, standard RMSE and MAPE are both used. For price direction detection, standard classification metrics are used. In this study, we use roc-auc and accuracy (which could be interpreted on a time range simulation into profitability measure).

\section{B. Raw Datasets}

The top popular cryptocurrencies with the highest market capitalizations are: Bitcoin (\$577.36), Ethereum (\$212.79), Tether (\$62.00), and Binance Coin (\$47.29) in Billions of dollars "https://coinmarketcap.com/all/views/all//" at the time of this writing. Error! Reference source not found. presents further information about datasets in hand.

TABLE I. TOP-TRADED CRYPTOCURRENCIES

\begin{tabular}{|c|c|l|}
\hline Crypto & Data date range & Samples \\
\hline Bitcoin (BTC) & $7 / 18 / 2010$ to $7 / 7 / 2021$ & 4000 \\
\hline Ethereum (ETH) & $3 / 10 / 2016$ to $7 / 7 / 2021$ & 1947 \\
\hline Tether (USDT) & $4 / 14 / 2017$ to $7 / 7 / 2021$ & 1546 \\
\hline Binance Coin (BNB) & $11 / 9 / 2017$ to $7 / 7 / 2021$ & 1338 \\
\hline
\end{tabular}

1) Bitcoin (BTC): Market cap: Over $\$ 641$ billion. Is the first cryptocurrency, founded in 2009 under the alias Satoshi Nakamoto [33]. Bitcoin's value has soared as it has grown in popularity. Five years ago, a Bitcoin could be purchased for around \$500. A single Bitcoin was worth more than $\$ 32,000$ in June 2021. This equates to a 6,300 percent increase. Plotting on Fig 2 visualizes Bitcoin data since its inception in 2010. In 2018, the price rose briefly before dropping dramatically in which is now known as bitcoin bubble (also known as the Bitcoin crash and the Great crypto crash). And in 2021, a much increasing tendency emerged.

2) Ethereum (ETH): Market cap: Over $\$ 307$ billion. Ethereum, which is both a cryptocurrency and a blockchain platform, is a favorite among programmers due to the potential applications it offers, such as smart contracts that execute automatically when certain conditions are met and nonfungible tokens (NFTs). Ethereum has also exploded in popularity. Its price increased by more than 22,000 percent in just five years, from around $\$ 11$ to over $\$ 2,500$.

3) Tether (USDT): Market cap: Over $\$ 62$ billion. Tether, unlike some other types of cryptocurrency, is a stable coin, which means it is backed by fiat currencies such as US dollars and the Euro and theoretically maintains a value equal to one 
of those denominations. In theory, this means that Tether's value should be more consistent than other cryptocurrencies, and it is preferred by investors who are wary of the extreme volatility of other coins.

4) Binance Coin (BNB): Market cap: Over $\$ 56$ billion. The Binance Coin is a type of cryptocurrency that can be used to trade and pay fees on Binance, one of the world's largest cryptocurrency exchanges. Binance Coin has grown beyond simply facilitating trades on Binance's exchange platform since its inception in 2017. It is now possible to use it for trading, payment processing, and even booking travel arrangements. It can also be traded or exchanged for other cryptocurrencies like Ethereum or Bitcoin. It was only $\$ 0.10$ in 2017; by June 2021, it had risen to over $\$ 350$, a gain of about 350,000 percent.

Each cryptocurrency dataset comprises of 5 indicators: open, low, high, close and volume. All data are acquired from https://www.investing.com API webservices via python library "investpy". investing.com is a global platform that delivers financial market analyses and news from across the world.

Besides historical cryptocurrencies datasets, other external factors datasets are used as shown in Error! Reference source not found.. Factors investigated in this study include:

1) Gold and Brent oil daily prices (open and volume values).
2) NYSE Bitcoin (NYXBT) index; the bitcoin index on the New York Stock Exchange. NYXBT indicates the US dollar value of one bitcoin unit based on actual transactions on selected bitcoin exchanges that have been assessed and meet NYSE quality criteria. The NYSE Bitcoin Index integrates data from Coinbase Exchange, the largest bitcoin exchange in the United States, in which NYSE has a minority investment [34].

3) The Standard and Poor's 500 or (SPX). SPX is a stock market index that tracks the performance of 500 big firms that are listed on US stock exchanges. As of December 31st, 2020, more than $\$ 4.6$ trillion has been invested in assets tied to the index's performance.

4) The Bitcoin and Ethereum Energy Consumption Index (PWR) is a measure of how much energy is consumed by the cryptocurrency mining in a certain time period available at "https://digiconomist.net". The data found online only available for Bitcoin and Ethereum.

5) Google Trends, https://trends.google.com/ . The daily historical data for two specific keywords: ("bitcoin", "Ethereum", "Tether", "Binance Coin", and "cryptocurrency") obtained from Google API webservices via the "pytrends" python module. Google Trends examines the popularity of top search queries in Google Search across several countries and languages. The website makes use of graphs to compare the search volume of various queries over time.
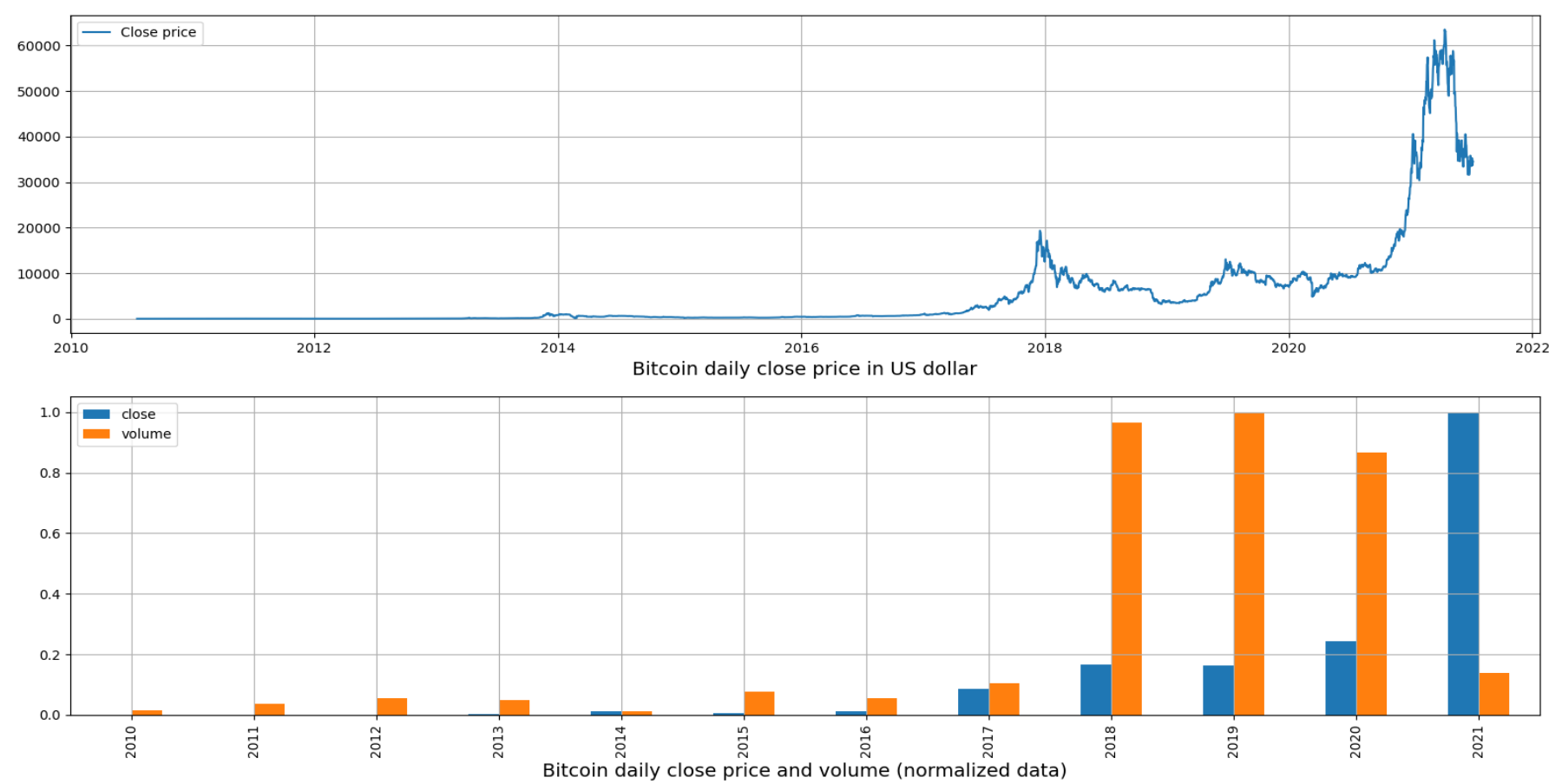

Fig. 2. Bitcoin Dataset Properties. 
TABLE II. EXTERNAL FACTORS

\begin{tabular}{|l|l|}
\hline Features & Description \\
\hline gold_close & Gold commodity material daily price \\
\hline oil_close & Brent oil daily price \\
\hline nyxbt & NYSE Bitcoin index \\
\hline spx & Standard and Poor's 500 \\
\hline pwr, pwr_min & $\begin{array}{l}\text { Bitcoin Energy Consumption Index (expected estimation } \\
\text { and minimum). }\end{array}$ \\
\hline gtrend_bitcoin & Google Trends for the cryptocurrency name keyword \\
\hline gtrend_crypto & Google Trends "cryptocurrency" keyword \\
\hline
\end{tabular}

\section{Features Engineering}

In this work, the strategy is to leverage the availability of various data sources as predictors for cryptocurrency price fluctuations and forecasting. In terms of which features are chosen for training, the features selection method is adaptive. The most important fundamental requirements are to find the right balance between training time and prediction accuracy. The proposed work dynamically infers the prediction power for each feature by using predictive power scoring (PPS) and correlation coefficient methods. PPS is an asymmetric, datatype agnostic tool for detecting linear and non-linear correlations between two columns. Values range from 1 (full predictive power) to zero (complete absence of predictive power). Asymmetric here in the sense that correlation between A-to-B is not the same as B-to-A. PPS uses a decision tree regression algorithm.

1) Features selection (Phase I): We use PPS, as a first phase filtration, to disregard features that have not much significance to the target prediction as PPS has the ability to detect non-linear relations.

2) Data preprocessing: We begin data preprocessing by normalizing the features. Normalization method is done by squashing the data from its original numerical scale to $[0,1]$ range. We make use of "MinMaxScaler" found in sklearn library. Normalization is essential step for neural networks innerworkings as unscaled data with higher values tend to wrongly dominate when calculating derivates by the optimizer. Also, normalization prevents some activation functions from getting numerically saturated. For detailed information please check [35]. Second, we devise a set of new fabricated features as following:

Crossing original features.

Encoding date minutes information.

Time lagging features.

3) Features Selection (Phase II): We continue the feature selection process by computing the linear statistical relationship between the target and candidate predictor variables with measures such as Pearson, Kendall, and Spearman. Pearson Correlation Coefficient (PCC) is used, which produces a value between $[-1,1]$, where an absolute value of one shows perfect correlation, the sign indicates whether the correlation is positive or negative, and zero indicates no linear dependency at all [36]. Fig 3 illustrates PPS and PCC analysis for bitcoin external features datasets. The target is the "close" price.

We think that the forecasting possibility may changes over time in a market as volatile and inefficient as cryptocurrency trading. To overcome this difficulty, it's necessary to adopt flexible features selection approach. We use an adaptive features selection strategy in where it selects the prospective features that have the best chance of being a good predictor throughout time.

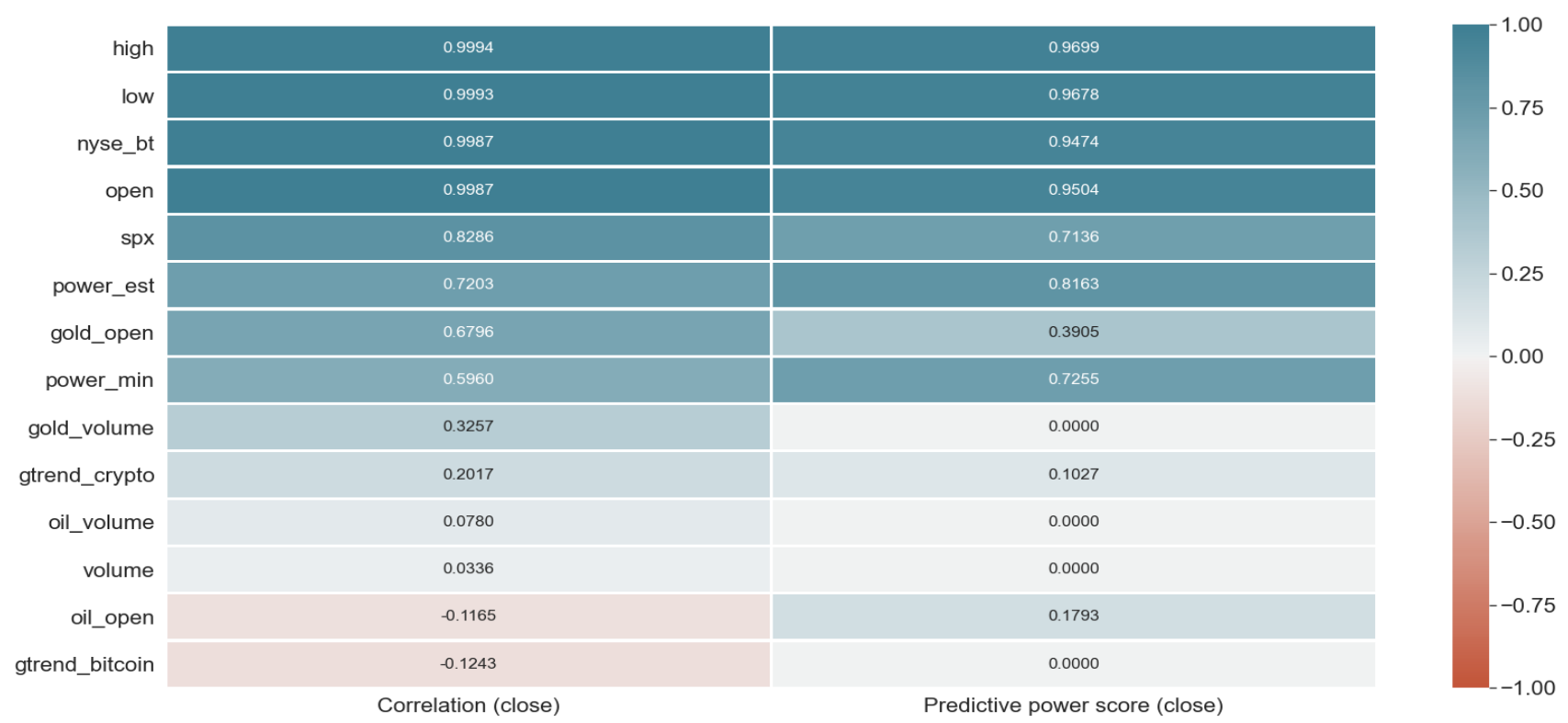

Fig. 3. Heat Map shows Correlation Coefficient and Predictive Power Scoring (Bitcoin External Features). 


\section{Proposed Model Architecture}

The proposed model is built around a deep learning engine that receives two tensors from a preprocessed multivariate timeseries datasets as input $\left(X_{p}\right.$ and $\left.X_{d}\right)$ where $X_{p}=$ $\left\{x_{1}^{p}, x_{2}^{p}, \ldots, x_{n}^{p}\right\}$ and $X_{d}=\left\{x_{1}^{d}, x_{2}^{d}, \ldots, x_{n}^{d}\right\}, n$ is the number of training samples. The target variables $\left(Y_{p}\right.$ and $\left.Y_{d}\right)$ are the cryptocurrency closing price value and direction $\mu$ which is encoded as $(1,-1)$ for up and down respectively. The prediction output vector $o=\left\{\hat{y}^{p}, \hat{y}^{d}\right\}$ where $\hat{y}^{p} \in \mathbb{R}$ is inferred from the learned reference distribution $p\left(Y^{p}\right) \cdot \hat{y}^{p}$ is a value which when scaled back to its original scale represents the forecasted closing price. $\hat{y}^{d}$ is further marginalized by a threshold $\varepsilon$ to label the closing price direction:

$\mu^{\text {sign }}=\left\{\begin{array}{c}\text { up if } \hat{y}^{d} \geq \varepsilon \\ \text { down if } \hat{y}^{d}<\varepsilon\end{array}\right.$

The proposed model consists of two stacks of layers for each input tensors $X_{p}$ and $X_{d}$ : 1) $X_{p}$ stack consists of three
GRU layers with 256 neurons each. Followed by MLP layer and an MLP output layer. 2) $X_{d}$ stack consists of three LSTM layer with 256 neurons each. Followed by MLP layer, dropout layer and an MLP output layer. As shown in Fig 4, output of $X_{p}$ stack GPU layer concatenates with $X_{d}$ stack LSTM layer and feeds the $X_{d}$ MLP layer. Layer counts, number of neurons per layer, batch sizes, training epochs, and other model hyperparameters are set empirically.

The training algorithm works as the following steps:

1) Step 1: the PPS values are computed for all raw inputs to identify $n$ factors with the largest predictability score. The target variable here is price "close".

2) Step 2: preprocessing features by normalizing data in range $[0,1]$ and devising new cross features.

3) Step 3: computing PCC values and further filter features to the selected $n$ features.

4) Step 4: train the neural network model on data.

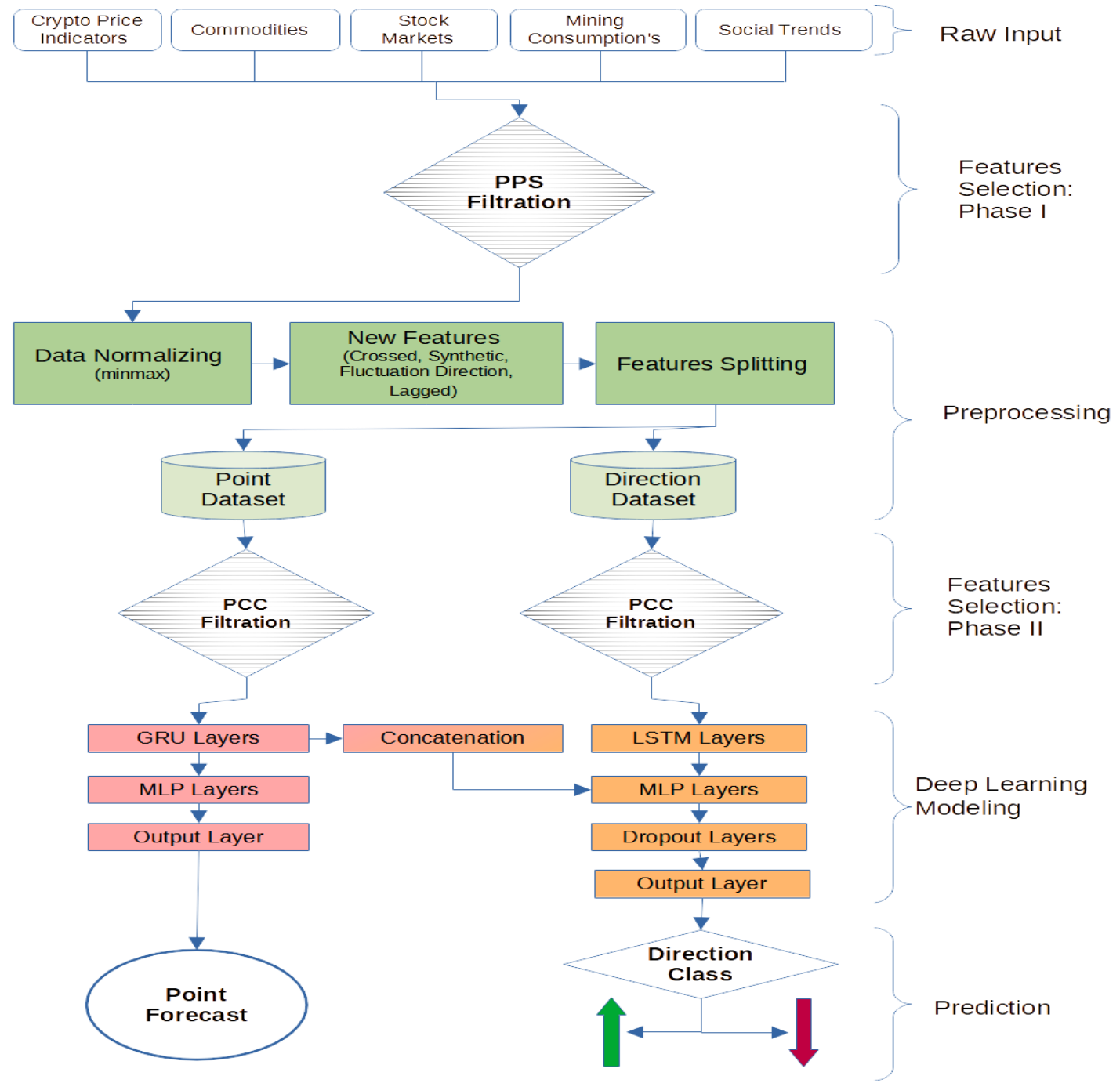

Fig. 4. System Diagram for the Proposed Cryptocurrency Model. 


\section{EXPERIMENTS AND RESULTS}

\section{A. Experiments Setup}

We implemented the proposed model on python 3.8 platform. The deep learning library is Keras on top of TensorFlow. Hardware consists of nVidia GTX 1660Ti (6GB dedicated RAM), 16GB RAM and $1710^{\text {th }}$ generation $2.6 \mathrm{GHz}$ processor. Results shown are the highest of multiple trials with different combination of configurations. Average training time within range $65_{-}^{+}$seconds with GPU accelerated computations. We split datasets in training/testing as $80 \%$ ratio.

For regression task, the model forecasts the price value of the next point. The regression branch of the model consists of two cascaded GRU layers of 256 neurons. Experiments show no benefit from applying recurrent dropouts. For the classification task of price movement direction, we trained the model with a list of two types of features: original and devised features.

\section{B. Results and Findings}

Results about bitcoin attained $92 \%$ accuracy score. We define accuracy as the overall proportion of correct predictions among the entire number of instances investigated. For regression, the proposed model achieved a score of 2.4 MAPE for bitcoin data. The overall performance metrics results are summarized in Error! Reference source not found.. Best classification achieved occurred with bitcoin data. As shown, the direction is successfully classified for all cryptocurrencies. Tether regression metrics are on top.

We can deduce no unique patterns or circumstances that are connected to the direction classification from the data. Fig 5 compares the classification of price movement direction to real data. The test data is shown in sub-figure (a) for the time period 3-2019 to 7-2021, with hits in blue circles and misses in red crosses. The data in sub-figure (b) is zoomed in to reveal one-month details. The true pricing data is shown by the green line.

TABLE III. RESULTS SUMMARY IN TERMS OF MAPE AND PR-AUC

\begin{tabular}{|l|l|l|l|l|l|}
\hline \multirow{2}{*}{ Cryptocurrency } & \multicolumn{2}{|l|}{ Regression } & \multicolumn{2}{l|}{ Classification } \\
\cline { 2 - 6 } & Mean $\mathbf{( \$ )}$ & RMSE $(\$)$ & MAPE $(\%)$ & Accuracy & PR-AUC \\
\hline Bitcoin (BTC) & 6690.87 & 850.8 & 2.28 & $92.1 \%$ & 0.981 \\
\hline Ethereum (ETH) & 425.6 & 82.03 & 3.17 & $90.26 \%$ & 0.930 \\
\hline Tether (USDT) & 0.999 & 0.0045 & 0.230 & $85.3 \%$ & $0.853 \%$ \\
\hline Binance Coin (BNB) & 56.4 & 26.7 & 3.100 & $63.29 \%$ & $0.743 \%$ \\
\hline
\end{tabular}

(a) Bitcoin test perfromance (classification)

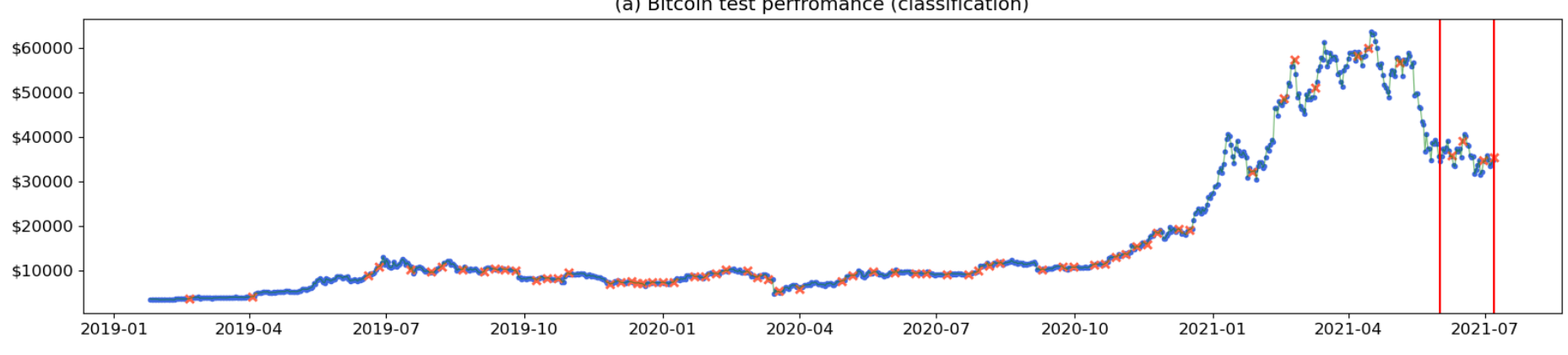

(b) Zoomed in (1 month)

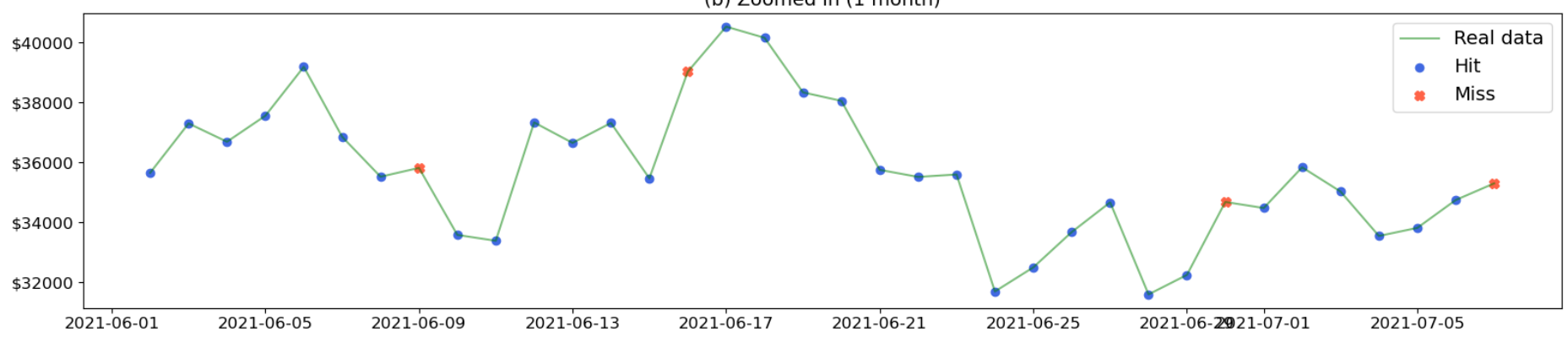

Fig. 5. Cryptocurrency Price Movement Classification Test Results (Hits and Misses). 
Regression results shown in Fig 6 are in orange color. RMSE for bitcoin is 850.8 . considering the scale of the bitcoin price which is extremely volatile from several thousands to tenth of thousands of dollars. RMSE don't reveal much about how good the regression results are. We can claim that MAPE is better representing the performance. In sub-figure (b), a one month zoom-in plotting illustrates the proposed model which performs a good job generalizing on unseen test data. In addition to forecast the next point, we forecast the $90 \%$ and $10 \%$ quantiles as a confidence boundaries (in sub-figure (b) as grey lines enclosing the real and forecasted values).

\section{Comparison with Related Work}

A Comparison with a three related deep learning work to the problem of interest reveals that the proposed model achieves highest accuracy in terms of classifying the next day price movement. From our perspective, adaptive features selection is a major contributor the results.

\section{Profitability Simulation}

We understand that traders must earn a profit at the end of the day. We use a basic trading algorithmic method to determine the projected profitability. We also use python code to implement it. The simulation method is naive since we simply want to illustrate how to utilize the anticipated results to prove the suggested model's profitability. Simulation results are shown as a waterflow chart in Fig 7. The simulation is based on the results of the tests ( 895 samples for bitcoin). The trade epochs were divided into 60 days period. The number of epochs is 15, with the first one beginning on March 3, 2019, and ending on July 7, 2021. The simulation resulted in 9 profits and 6 losses. The profit was 199038 dollars, with a return of 68.24 percent, on a maximum investment of 291690 dollars. Over time, the volume of profit and losses represents the rising and diminishing volatility of cryptocurrency trading prices and volumes, with the biggest earnings and losses accompanying the most spectacular jumps and falls, respectively.

\section{E. Final Discussion}

The predictive power score (PPS) method is used in Phase I of the feature selection procedure to exclude features that have little potential for cryptocurrency prediction. When using PPS, there are two variables to consider: 1) the threshold, which is the PPS score value used to determine if a feature is acceptable or not. 2) The effective sample size, which is the number of samples needed to compute the PPS score, or the cryptocurrency time period over which we are looking for the best predictors. The effect of these two factors on MAPE and accuracy is depicted in Fig 8. When we ignore predictors with a score of less than 0.3 , as shown in sub-figure (a), the least regression MAPE value happens. In sub-figure (b), a score of 0.5 results in the most accurate price movement direction classification. When we limit the effective sample size to the last 500 samples of the training data in (c), we get the lowest MAPE value, and when we increase the size to 1000 samples, we get the maximum accuracy as shown in (d).

TABLE IV. COMPARISON WITH RELATED WORK

\begin{tabular}{|l|l|l|}
\hline Model & Technique & Accuracy \\
\hline $\begin{array}{l}\text { RNN-based ensemble } \\
{[37]}\end{array}$ & $\begin{array}{l}\text { RNN-based ensemble with decision } \\
\text { tree classifier }\end{array}$ & $62.91 \%$ \\
\hline CNN-BiLSTM [38] & $\begin{array}{l}\text { Convolutional-based bidirectional } \\
\text { LSTM }\end{array}$ & $55.43 \%$ \\
\hline $\begin{array}{l}\text { Bayesian optimized } \\
\text { LSTM [39] }\end{array}$ & Bayesian optimization LSTM & $76.83 \%$ \\
\hline The proposed model & $\begin{array}{l}\text { RNN-based with adaptive features } \\
\text { selection }\end{array}$ & $\mathbf{9 3 . 1 \%}$ \\
\hline
\end{tabular}

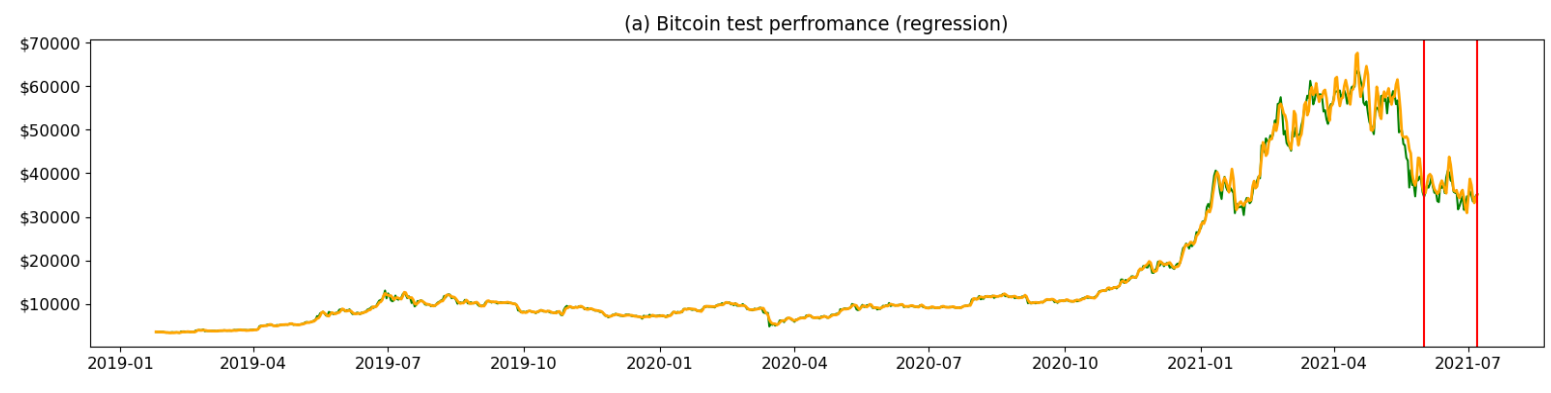

(b) Zoomed in (1 month)

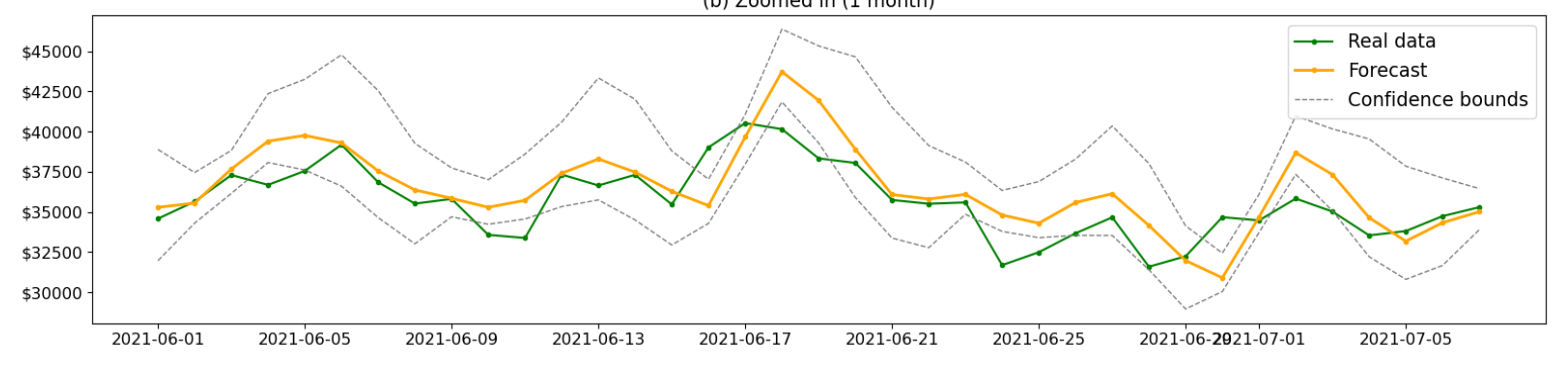

Fig. 6. Cryptocurrency Price Forecast Results (Next Point Forecast). 


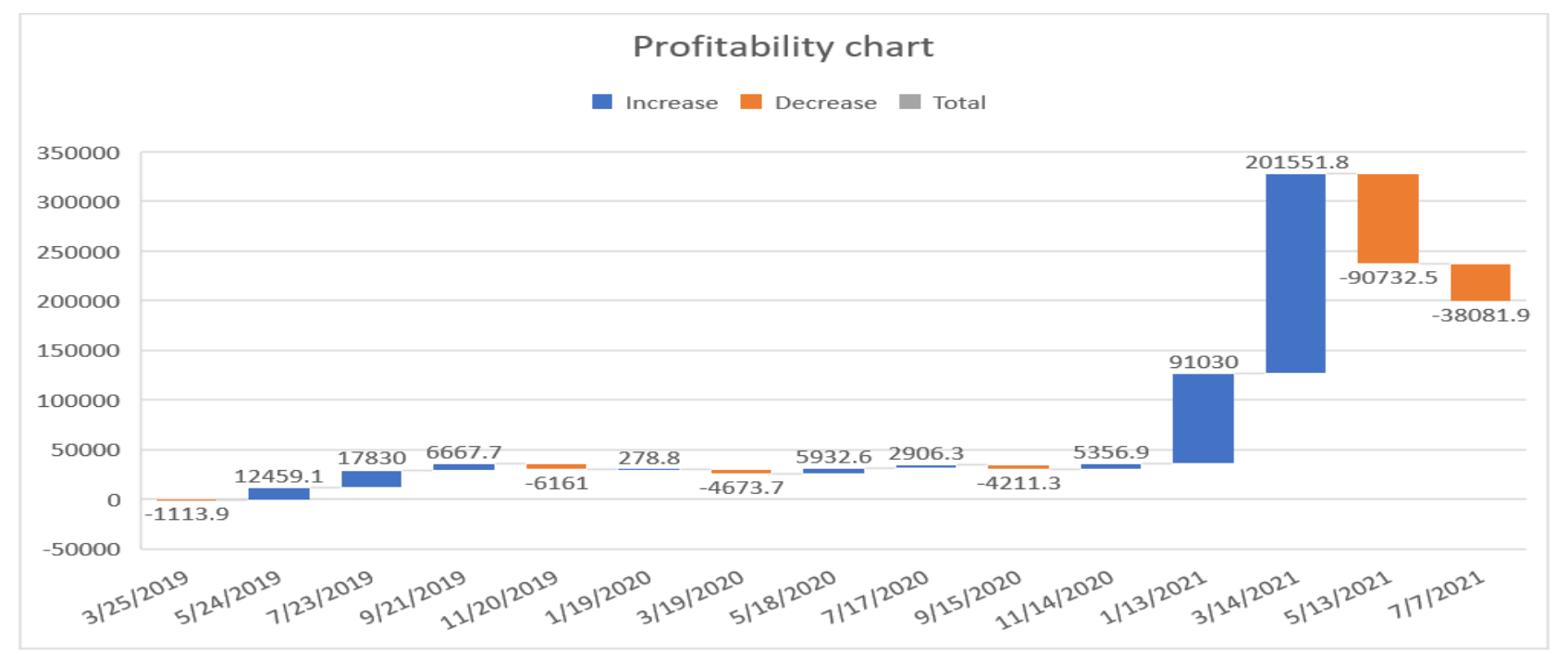

Fig. 7. Profitability Simulation Results.

The model's ability to forecast and classify accurately is influenced by the values of recurrent timesteps. Formally, the model needs to learn the probability of a sample $y_{i+1}$ at time $i+1$, given the context $x_{i-t: i}$ of earlier $t$ input features samples: $p\left(y_{i+1} \mid x_{i-t: i}\right)$. RNN (LSTM, GRU, and other recurrent neural network variants) incorporate past data in addition to the current data point, which is the timestep parameter $t$ passed to the RNN layers. Contextual-based prediction increases model performance. We may consider that point inside the problem of interest as follows: we use the earlier $n$ days to properly forecast the price value and direction of the next day. $\widehat{y_{l}}=\operatorname{model}\left(X_{i-t}^{i}\right)$, where $\widehat{y_{l}}$ is the model prediction at point $i, X_{i-t}^{i}$ input vector of $t$ number of samples prior to $i$ time, $t \in \mathbb{N}$ is the timesteps parameter. Sub-figures (a) and (b) shows model behavior with different timesteps values. Apparently, a timestep value of "7" gives lowest MAPE and highest accuracy.

The predictors vector is shifted back into the time domain using time lags. $\operatorname{lag}_{l}=x_{t-l}$, where $l \in \mathbb{N}$ is the lag value, $t$ is the time point. As an example, $l a g_{1}$ is using the previous day $t-1$ to predict the coming day $t$. $\operatorname{lag}_{2}$ is using the day before $t-2$ to predict the coming day $t$. Usually in seasonal data with high stationarity, autocorrelation function (ACF) analysis computes which time lag is best to be used as a predictor. In addition, in timeseries forecasting lags could be looked in as the forecast horizon. However, in case in hand, the situation is different since cryptocurrency timeseries data is a random walk and non-stationary, i.e., no seasonality, no steady statistical moments values and high variability. Augmented Dickey Fuller Test (ADF Test) on bitcoin "close" data give a p-value of " 0.738698 " which can be interpreted as strong nonstationarity property $(p-$ value $>0.05)$. Fig 9 shows time lags from " 1 " to "7", $\operatorname{lag}_{1}$ gives best performance. also, we notice accuracy keeps above $60 \%$ till $\operatorname{lag}_{5}$, that means profitability still possible till forecasting horizon of 5-days (we limit the scope to daily data, forecasting long-term periods such as months requires a different data frequencies and different strategies).

In deep learning, hyper-parameters tuning task requires a lot of hands on efforts, and sometimes the task is automated [40]. Fig 10 depicts two model hyper-parameters: neurons per recurrent layers and dropouts. As we can notice, for regression layers, a "128" gives least MAPE and for classification, a "256" neurons give highest accuracy. For both tasks, a dropout of 0.1 is enough to have a positive impact on the predictions.

: 1) Are cryptocurrency prices predictable? And if so, is that due to inherent features of the pricing data? or as a result of external influences? 2) Which is more important in terms of profitability: predicting the next price value or predicting the future price direction (increase or drop) over a forecasting horizon? 3) Can deep learning algorithms accurately forecast the price of cryptocurrencies?

We have presented applied research to address both problems in cryptocurrency prices fluctuations, the next point forecast and next price movement direction classification. We have included several features grouped into cryptocurrency indicators ("open", "close", "low", "high" and "volume") and external factors gathered from various sources, including "SPX", "NYCE_BT", "GTrends_CRYPTO", and others.

The results of the experiments and the profitability simulation done in this study show that we can develop predictive model for two tasks using deep learning methods, particularly, recurrent neural networks algorithms: regression of the future pricing values and classification of the direction of price movement. A careful features selection strategy is mandatory to successfully identify good predictors and how to include them in learning process. Such results answer the third research question: "Can deep learning methods accurately forecast the price of cryptocurrencies and classify its movement direction?" 

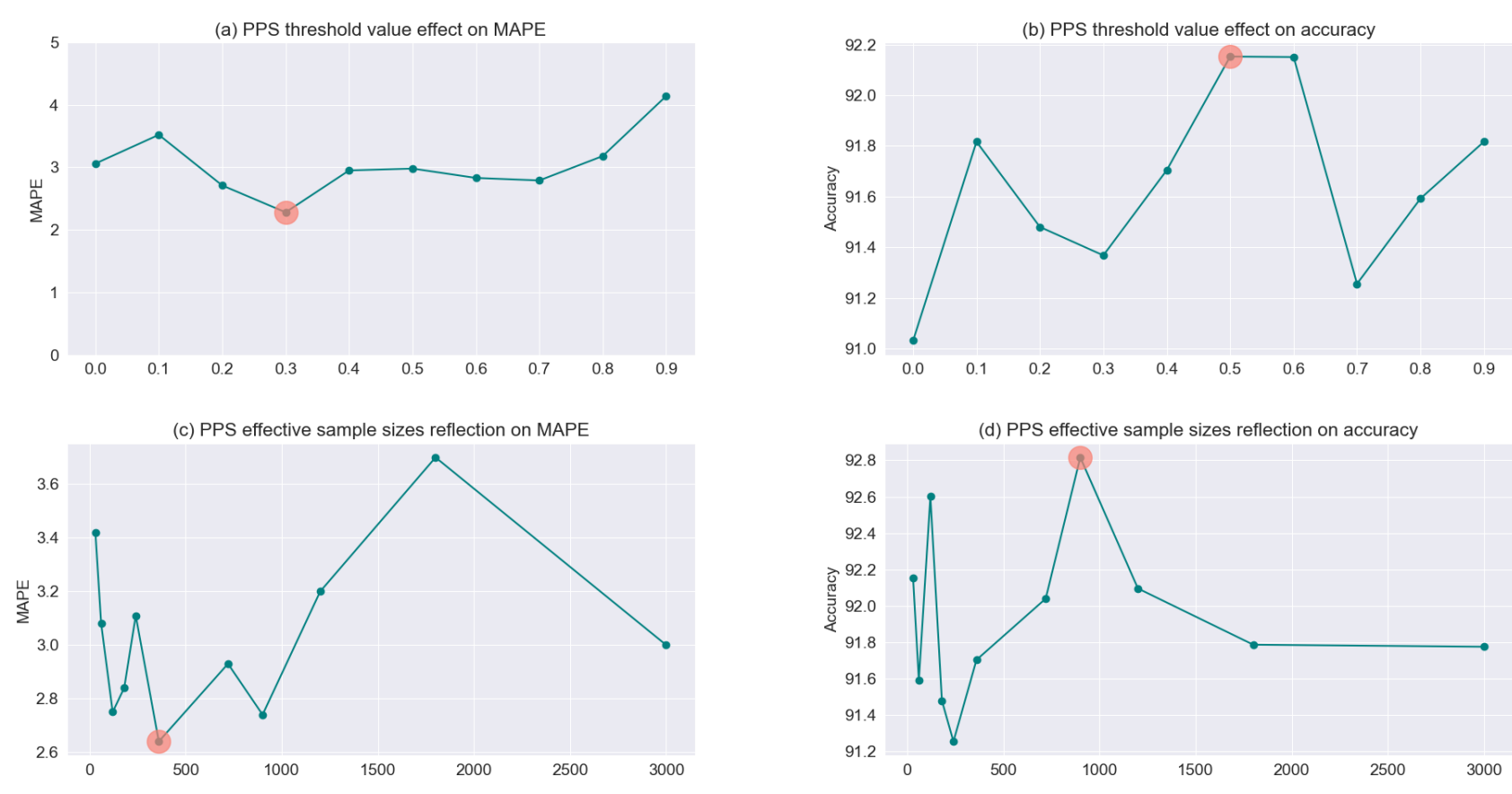

Fig. 8. PPS Settings Effect on Accuracy and MAPE.
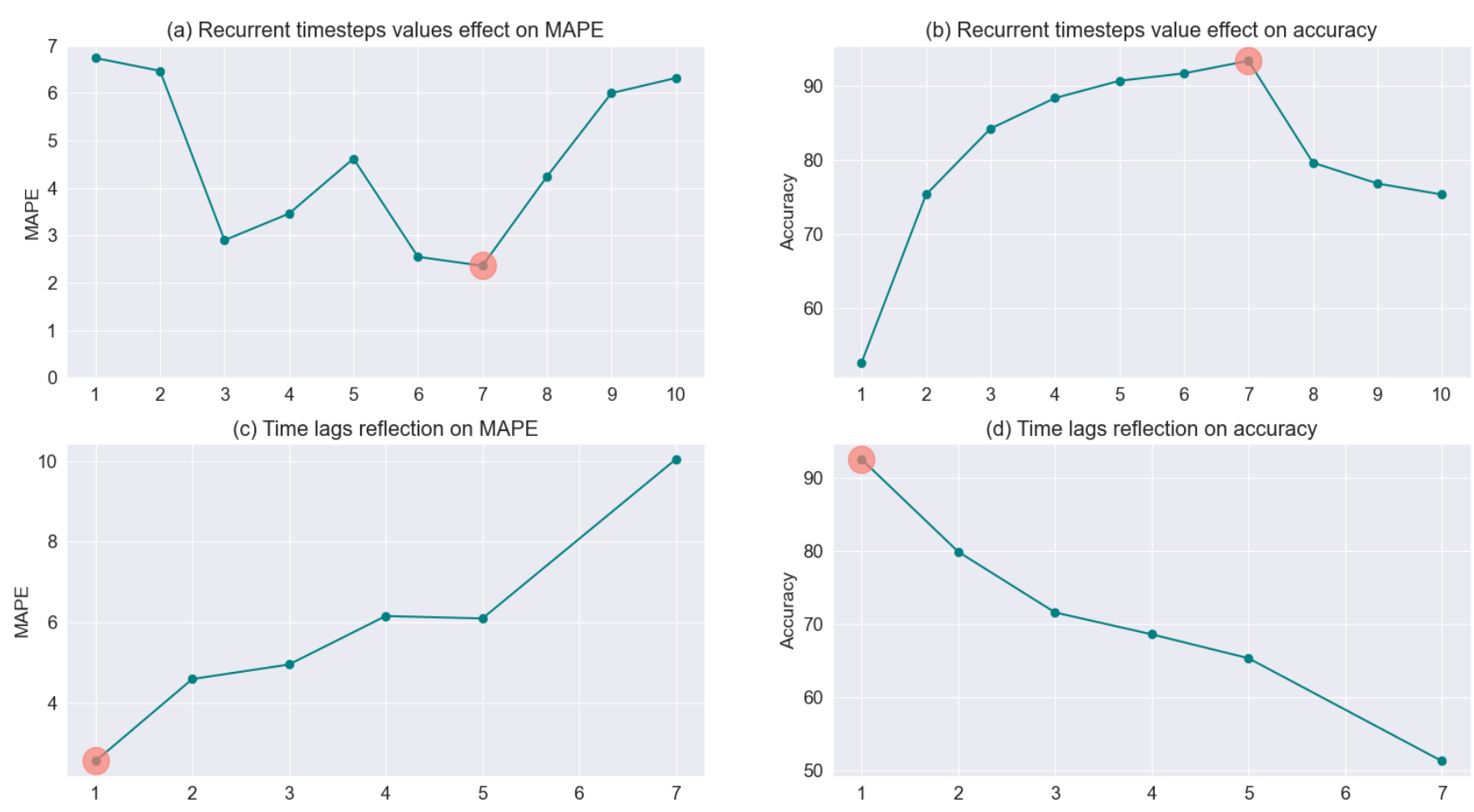

Fig. 9. Timesteps and Forecasting Lags Effect on Performance. 


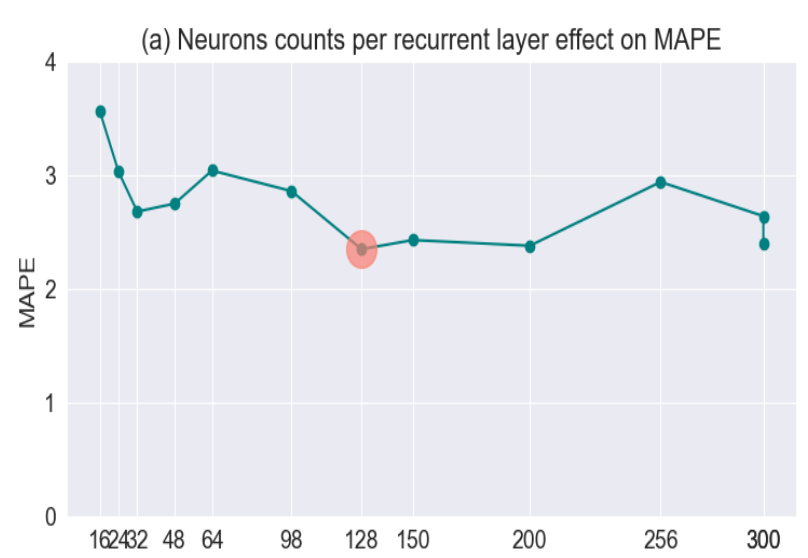

(c) Droupout values reflection on MAPE

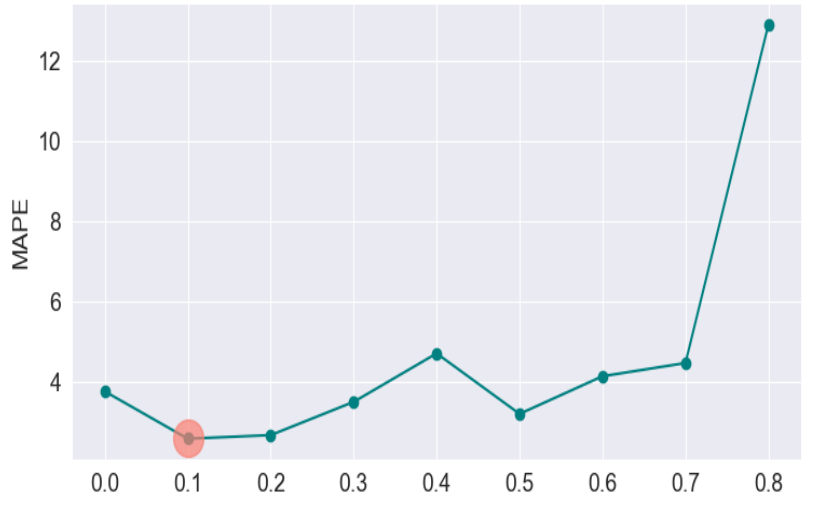

(b) Neurons counts per recurrent layer effect on accuracy

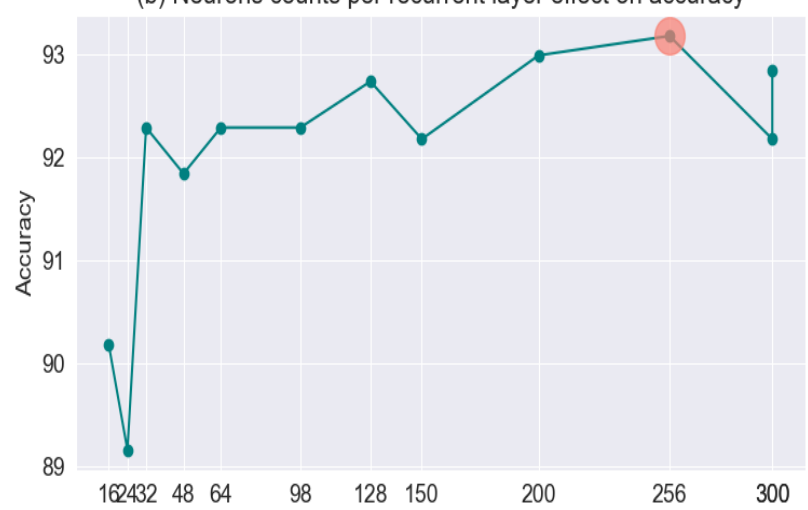

(d) Droupout values reflection on accuracy

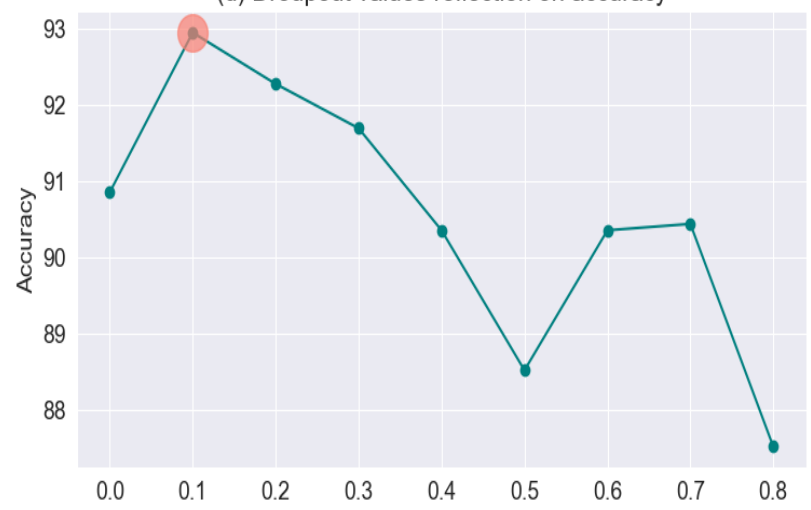

Fig. 10. Units and Dropouts Parameters Effect on Performance.

\section{CONCLUSIONS AND FUTURE WORK}

This research presents a deep neural network model for bitcoin price/movement prediction based on a multi-input architecture. To determine which variables to include, a twophase adaptive feature selection process is applied. As a result of this method, the data provided to the neural network has the maximum predictive power for the price. The results suggest that using an adaptive feature selection technique to improve classification performance has a significant influence. Future study could consider using more advanced deep learning architectures, such as attention mechanisms, to focus on key patterns in data. Another idea is to look at generative adversarial architectures for various speculative scenarios with varying levels of confidence. In addition, other external elements that correlate or have a degree of predictability with the bitcoin price movement should be included.

\section{REFERENCES}

[1] J. Chu, S. Chan, S. Nadarajah, and J. Osterrieder, "GARCH Modelling of Cryptocurrencies,” J. Risk Financ. Manag., vol. 10, no. 4, p. 17, 2017, doi: $10.3390 / \mathrm{jrfm} 10040017$.

[2] P. Katsiampa, "An empirical investigation of volatility dynamics in the cryptocurrency market," Res. Int. Bus. Financ., vol. 50, pp. 322-335, 2019, doi: 10.1016/j.ribaf.2019.06.004.

[3] A. S. Hayes, "Cryptocurrency value formation: An empirical study leading to a cost of production model for valuing bitcoin," Telemat. Informatics, vol. 34, no. 7, pp. 1308-1321, 2017, doi: 10.1016/j.tele.2016.05.005.
[4] B. G. Malkiel, "The efficient market hypothesis and its critics," J. Econ. Perspect., vol. 17, no. 1, pp. 59-82, 2003.

[5] S. Palamalai, K. K. Kumar, and B. Maity, "Testing the random walk hypothesis for leading cryptocurrencies," Borsa Istanbul Rev., 2020, doi: 10.1016/j.bir.2020.10.006.

[6] C. Peng and G. Yichao, "Cryptocurrency Price Analysis and Time Series Forecasting," no. April, 2020.

[7] R. K. Malladi and P. L. Dheeriya, "Time series analysis of Cryptocurrency returns and volatilities," J. Econ. Financ., vol. 45, no. 1, pp. 75-94, 2021, doi: 10.1007/s12197-020-09526-4.

[8] B. M. Blau, "Price dynamics and speculative trading in bitcoin," Res. Int. Bus. Financ., vol. 41, pp. 493-499, 2017.

[9] N. A. Bakar and S. Rosbi, "Autoregressive Integrated Moving Average (ARIMA) Model for Forecasting Cryptocurrency Exchange Rate in High Volatility Environment: A New Insight of Bitcoin Transaction," Int. J. Adv. Eng. Res. Sci., vol. 4, no. 11, pp. 130-137, 2017, doi: 10.22161/ijaers.4.11.20.

[10] C. G. Akcora, A. K. Dey, Y. R. Gel, and M. Kantarcioglu, "Forecasting bitcoin price with graph chainlets," Lect. Notes Comput. Sci. (including Subser. Lect. Notes Artif. Intell. Lect. Notes Bioinformatics), vol. 10939 LNAI, pp. 765-776, 2018, doi: 10.1007/978-3-319-93040-4_60.

[11] C. G. Akcora, M. F. Dixon, Y. R. Gel, and M. Kantarcioglu, "Bitcoin risk modeling with blockchain graphs," Econ. Lett., vol. 173, pp. 138142, 2018, doi: 10.1016/j.econlet.2018.07.039.

[12] A. K. Dey, C. G. Akcora, Y. R. Gel, and M. Kantarcioglu, "On the role of local blockchain network features in cryptocurrency price formation," Can. J. Stat., vol. 48, no. 3, pp. 561-581, 2020, doi: 10.1002/cjs.11547.

[13] V. Derbentsev, V. Babenko, K. Khrustalev, H. Obruch, and S. Khrustalova, "Comparative performance of machine learning ensemble algorithms for forecasting cryptocurrency prices," Int. J. Eng. Trans. A Basics, vol. 34, no. 1, pp. 140-148, 2021, doi: 10.5829/IJE.2021.34.01A.16. 
[14] M. M. Patel, S. Tanwar, R. Gupta, and N. Kumar, "A Deep Learningbased Cryptocurrency Price Prediction Scheme for Financial Institutions," J. Inf. Secur. Appl., vol. 55, no. May, 2020, doi: 10.1016/j.jisa.2020.102583.

[15] K. Wołk, "Advanced social media sentiment analysis for short-term cryptocurrency price prediction," Expert Syst., vol. 37, no. 2, pp. 1-16, 2020, doi: 10.1111/exsy.12493.

[16] J. Abraham, D. Higdon, and J. Nelson, "Cryptocurrency price prediction using tweet volumes and sentiment analysis," SMU Data Sci. Rev., vol. 1 , no. 3, p. 22, 2018, [Online]. Available: https://scholar.smu.edu/datasciencereviewhttp://digitalrepository.smu.ed u.Availableat:https://scholar.smu.edu/datasciencereview/vol1/iss3/1.

[17] D. Shen, A. Urquhart, and P. Wang, "Does twitter predict Bitcoin?," Econ. Lett., vol. 174, pp. 118-122, 2019, doi: 10.1016/j.econlet.2018.11.007.

[18] S. Mohapatra, N. Ahmed, and P. Alencar, "KryptoOracle: A Real-Time Cryptocurrency Price Prediction Platform Using Twitter Sentiments," Proc. - 2019 IEEE Int. Conf. Big Data, Big Data 2019, pp. 5544-5551, 2019, doi: 10.1109/BigData47090.2019.9006554.

[19] S. Bhambhwani, S. Delikouras, G. M. Korniotis, and others, Do fundamentals drive cryptocurrency prices? Centre for Economic Policy Research, 2019.

[20] H. Bystrom and D. Krygier, "What Drives Bitcoin Volatility?," SSRN Electron. J., 2018, doi: 10.2139/ssrn.3223368.

[21] P. Giudici and I. Abu-Hashish, "What determines bitcoin exchange prices? A network VAR approach," Financ. Res. Lett., vol. 28, pp. 309318, 2019.

[22] L. dos Santos Maciel and R. Ballini, "On the predictability of high and low prices: The case of Bitcoin,” Rev. Bras. Finanças, vol. 17, no. 3, pp. 66-84, 2019.

[23] V. Derbentsev, N. Datsenko, O. Stepanenko, and V. Bezkorovainyi, "Forecasting cryptocurrency prices time series using machine learning," CEUR Workshop Proc., vol. 2422, pp. 320-334, 2019.

[24] A. Greaves and B. Au, "Using the Bitcoin Transaction Graph to Predict the Price of Bitcoin," pp. 1-8, 2015.

[25] N. A. Hitam, A. R. Ismail, and F. Saeed, "An Optimized Support Vector Machine (SVM) based on Particle Swarm Optimization (PSO) for Cryptocurrency Forecasting," Procedia Comput. Sci., vol. 163, pp. 427433, 2019, doi: 10.1016/j.procs.2019.12.125.

[26] P. Mohanty, D. Patel, P. Patel, and S. Roy, "Predicting Fluctuations in Cryptocurrencies' Price using users' Comments and Real-time Prices," 2018 7th Int. Conf. Reliab. Infocom Technol. Optim. Trends Futur. Dir. ICRITO 2018, no. August, pp. 477-482, 2018, doi: 10.1109/ICRITO.2018.8748792.

[27] A. Mittal, V. Dhiman, A. Singh, and C. Prakash, "Short-term bitcoin price fluctuation prediction using social media and web search data," in
2019 Twelfth International Conference on Contemporary Computing (IC3), 2019, pp. 1-6.

[28] G. S. Atsalakis, I. G. Atsalaki, F. Pasiouras, and C. Zopounidis, "Bitcoin price forecasting with neuro-fuzzy techniques," Eur. J. Oper. Res., vol. 276, no. 2, pp. 770-780, 2019, doi: 10.1016/j.ejor.2019.01.040.

[29] I. A. Hashish, F. Forni, G. Andreotti, T. Facchinetti, and S. Darjani, "A hybrid model for bitcoin prices prediction using hidden Markov models and optimized LSTM networks," in 2019 24th IEEE International Conference on Emerging Technologies and Factory Automation (ETFA), 2019, pp. 721-728.

[30] A. Radityo, Q. Munajat, and I. Budi, "Prediction of Bitcoin exchange rate to American dollar using artificial neural network methods," in 2017 International Conference on Advanced Computer Science and Information Systems (ICACSIS), 2017, pp. 433-438.

[31] M. Maiti, Y. Vyklyuk, and D. Vuković, "Cryptocurrencies chaotic comovement forecasting with neural networks," Internet Technol. Lett., vol. 3, no. 3, pp. 1-6, 2020, doi: 10.1002/it12.157.

[32] M. Iqbal, M. S. Iqbal, F. H. Jaskani, K. Iqbal, and A. Hassan, "TimeSeries Prediction of Cryptocurrency Market using Machine Learning Techniques," EAI Endorsed Trans. Creat. Technol., p. e4, 2021.

[33] S. Nakamoto, "Bitcoin: A peer-to-peer electronic cash system," Decentralized Bus. Rev., p. 21260, 2008.

[34] Treasurers.org, "NYSE launches Bitcoin Index," https://www.treasurers.org/, 2021. https://www.treasurers.org/hub/ treasurer-magazine/nyse-launches-bitcoin-index (accessed Jul. 07, 2021).

[35] X. Wan, "Influence of feature scaling on convergence of gradient iterative algorithm," J. Phys. Conf. Ser., vol. 1213, no. 3, 2019, doi: 10.1088/1742-6596/1213/3/032021.

[36] J. Benesty, J. Chen, Y. Huang, and I. Cohen, "Pearson correlation coefficient," in Noise reduction in speech processing, Springer, 2009, pp. $1-4$.

[37] D. C. A. Mallqui and R. A. S. Fernandes, "Predicting the direction, maximum, minimum and closing prices of daily Bitcoin exchange rate using machine learning techniques," Appl. Soft Comput. J., vol. 75, pp. 596-606, 2019, doi: 10.1016/j.asoc.2018.11.038.

[38] P. Pintelas, T. Kotsilieris, I. Livieris, E. Pintelas, and ..., "Fundamental research questions and proposals on predicting cryptocurrency prices using DNNs," 2020, [Online]. Available: http://nemertes.lis.upatras.gr/jspui/handle/10889/13296.

[39] M. Rahmani Cherati, A. Haeri, and S. F. Ghannadpour, "Cryptocurrency direction forecasting using deep learning algorithms," J. Stat. Comput. Simul., 2021, doi: 10.1080/00949655.2021.1899179.

[40] A. S. Elberawi and others, "A Deep Learning Approach for Forecasting Global Commodities Prices," Futur. Comput. Informatics J., vol. 6, no. 1, p. 4, 2021. 